\title{
A Model for Electrcal Stimulation of Central Myelinated Fibers with Monopolar Electrodes
}

\author{
Spencer L. BeMent and James B. Ranck. Jr. ${ }^{1}$ \\ Bioelectric Scicnces Laboratory, Department of Elcctrical Engineering; and \\ Department of Physiology', The Unicersity of Michigan, \\ Ann Arbor, Michigan $4810+$
}

Reccir'ed January 29, 1969

\begin{abstract}
A mathematical model for monopolar cathodal stimulation of myelinated fibers in an anisotropic medium is developed. This model includes all of the factors known to affect significantly the stimulation properties of myelinated fibers. The quantitative relationship between stimulation current and electrode distance predicted by the model compares favorably with experimental data for stimulating electrode distances greater than about $500 \mu$. The model is used to predict electrical stimulation properties for myelinated fibers in other regions of the central nervous system.
\end{abstract}

\section{Introduction}

The bulk of the experimental results presented in the previous paper (2a) are for the fairly restrictive case of monopolar cathodal stimulation of single myelinated fibers in an anisotropic medium by rectangular currents of $50-\mu \mathrm{sec}$ duration from a $100-\mu$ diameter platinum-iridium electrode located at the surface of the medium. In this paper, a quantitative model is developed which can explain much of the experimental data on the basis of seemingly reasonable assumptions. The model is composed of all the factors usually thought to determine the electrical stimulation properties of myelinated fibers. Furthermore, we shall show how this model can be used to predict stimulation characteristics for myelinated fibers in situations beyond those directly measured, even when absolute values of all the parameters in the model are not known accurately.

The stimulation situation for a monopolar cathodal electrode located directly above a node of Ranvier is depicted in Fig. 1. The electrode diameter, the distance of the electrode from the fiber, and the internodal length are drawn to approximate their relative relationships. However, for the sake of clarity, the fiber diameter and nodal width are greatly exaggerated.

1. This work was supported in part by a research grant from the National Institute of Neurological Diseases and Blindness (NB-04352) and by the Horace H. Rackham Endowment. 


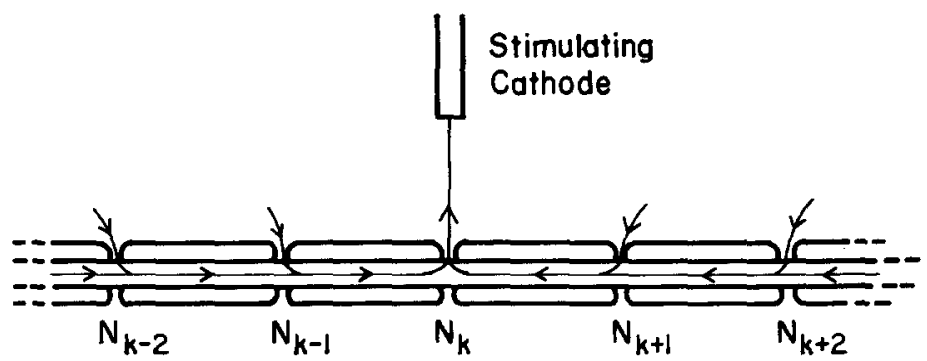

FIG. 1. A schematic representation of cathodal current flow lines for a stimulating electrode located directly above a myelinated fiber node. In actuality, current may be flowing out across nodes immediately adjacent to the stimulated node as well.

The model developed is based upon the solution to the difference equation generated from the usual lumped equivalent circuit for a section of an infinitely long cylindrical myelinated fiber. The assumptions associated with Fig. 2 and with the solution to the difference equation are listed below. A list of symbols related to Fig. 2 and to the development of the quantitative model is also given.

\section{Assumptions}

1. The geometry of the system is represented by a group of infinitely long parallel cylinders with conducting cores and surface membranes, and a common external conducting layer, all with linear electrical characteristics.

2. All of the currents are zero and the potential is constant throughout the core and external fluid at any point along the length of the model when the fiber is in the resting state and there is no outside current.

3. The internal and external fluids represent pure constant linear resistances.

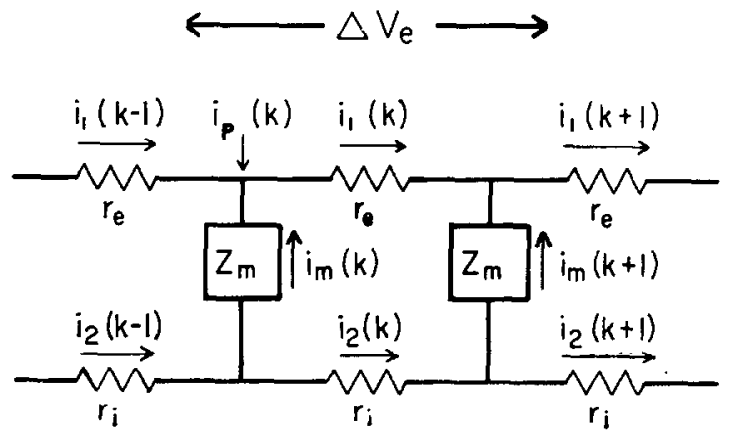

FIG. 2. An equivalent circuit for a representative portion of a myelinated fiber. For nomenclature see the text. 
4. The excited nerve fiber acts in a nuanner similar to the theoretical model of Hodgkin and Huxley (10). Thus the membranes can be considered as equivalent to a membrane capacitance in parallel with series combinations of membrane ion resistances and ion potentials.

5. The myelin is a perfect insulator, broken only at equally spaced intervals by nodes of infinitesimal length and finite resistance.

6 . The node where an action potential is first generated is directly below the cathode.

Assumptions 1-5 are basically the same as those used by Hodgkin and Rushton (11), Taylor (24), and Patlak (16). Assumption 6 simplifies the derivation of the quantitative model without any loss of generality, as will be apparent later. The other assimptions necessary to make the model quantitative are introduced at various points in the development. A detailed discussion of these last assumptions follows the derivation of the quantitative model.

\section{TABLE 1}

List of SyMbols

$r_{\mathrm{c}}=$ Resistance per centimeter of the external fluid (ohms $\left./ \mathrm{cm}\right)$.

$r_{\mathrm{i}}=$ Resistance per centimeter of the inner core of a fiber (ohms/cm).

$R_{m}=$ Resistance of a node (ohms).

$C_{\mathrm{m}}=$ Capacitance of a node (farads).

$Z_{\mathrm{m}}=$ Complex impedance of a node (ohms).

$i_{1}=$ Longitudinal current flowing in the external fluid (amp).

$i_{2}=$ Longitudinal current flowing in the inner core of a fiber (amp).

$i_{\mathrm{m}}=$ Current flowing outward across the nodal membrane $(\mathrm{amp} / \mathrm{cm}) .{ }^{2}$

$i_{1}=$ Current per centimeter length flowing radially into the outer fluid from an outside electrode $(\mathrm{amp} / \mathrm{cm})$.

$I_{n}=$ Transverse current flowing outward across a node (amp).

$I_{\mathrm{p}}=$ Total current through the electrodes (amp).

$V_{\mathrm{c}}=$ Voltage between two successive nodes $(v)$.

$V_{\mathrm{m}}=$ Change in voltage across the nodal membrane when $I_{\mathrm{m}}$ is flowing (v).

$r=$ Axis cylinder radius $(\mu)$.

$K=$ A constant which relates the axis cylinder radius to the internodal length.

$l=$ Distance between nodes $(\mathrm{cm})$.

$n=$ Number of fibers in the surrounding medium.

2 The convention of im as an imitard current was used by Taylor (24) and Patlak (16). However, the modern convention is to define $i_{\mathrm{m}}$ as a depolarizing current which is directed outward across the node. 


\section{Model Development}

Figure 2 indicates an equivalent circuit for a representative portion of a myelinated fiber and is usually designated as the one-dimensional or simple core conductor model. In a manner completely analogous to that of Taylor (24) and Patlak (16), one finds the difference equation

$I_{\mathrm{m}}(k+1)-\left[2+l\left(n r_{\mathrm{e}}+r_{\mathrm{j}}\right) / Z_{\mathrm{m}}\right] I_{\mathrm{m}}(k)+I_{\mathrm{m}}(k-1)=l r_{\mathrm{c}} i_{\mathrm{p}}(k) / Z_{\mathrm{m}}$,

when a fiber is stimulated by an infinitesimally small monopolar current electrode located at the $k^{\mathrm{lu}}$ node. The solution given by Patlak for $i_{\mathrm{p}}(k)=0$ when $k \neq 0$, and $i_{\mathrm{p}}(\mathrm{k})=I_{\mathrm{p}}$ when $k=0$ is

$$
I_{\mathrm{m}}(0)=\frac{r_{\mathrm{e}}}{\left(n r_{\mathrm{e}}+r_{\mathrm{i}}\right)\left[1+\frac{4 Z_{\mathrm{m}}}{\left(n r_{\mathrm{e}}+r_{\mathrm{i}}\right)}\right]^{\frac{1}{2}}} I_{\mathrm{p}} .
$$

The solution of the difference equation as represented by Eq. [2] involves an assumption that the extracellular potential field is linear with distance in the direction of the fiber. This important assumption is justified for distances greater than about $400 \mu$ in the discussion associated with Fig. 4.

Summation of the currents at the $k=0$ node gives

$$
I_{\mathrm{p}}(0)+i_{1}(-1)-i_{1}(0)+I_{\mathrm{m}}(0)=0 .
$$

However, from the symmetry of the fiber with respect to the stimulating electrode

$$
i_{1}(-1)=-i_{1}(0)
$$

Therefore,

$$
i_{1}(0)=\left[I_{\mathrm{p}}+I_{\mathrm{m}}(0)\right] / 2 .
$$

Equation 2 is solved for $I_{\mathbf{p}}$ and substituted into Eq. [5] along with $I_{\mathrm{m}}(0)=V_{\mathrm{m}}(0) / Z_{\mathrm{m}}$ to give

$$
i_{1}(0)=\frac{V_{\mathrm{m}}(0)}{2 Z_{\mathrm{m}}}\left[\frac{\left(n r_{\mathrm{e}}+r_{\mathrm{i}}\right)}{r_{\mathrm{e}}}\left(1+\frac{4 Z_{\mathrm{m}}}{\left(n r_{\mathrm{e}}+r_{\mathrm{i}}\right)}\right)^{\frac{1}{2}}+1\right] .
$$

Therefore,

$\Delta V_{\mathrm{e}}=i_{1}(0) l r_{\mathrm{e}}=\frac{V_{\mathrm{m}}(0) l r_{\mathrm{e}}}{2 Z_{\mathrm{m}}}\left[\frac{\left(n r_{\mathrm{e}}+r_{\mathrm{i}}\right)}{r_{\mathrm{e}}}\left(1+\frac{4 Z_{\mathrm{m}}}{l\left(n r_{\mathrm{e}}+r_{\mathrm{i}}\right)}\right)^{\frac{1}{2}}+1\right]$.

If $R_{\mathrm{m}}=4 l\left(n r_{\mathrm{e}}+r_{\mathrm{i}}\right)$ as discussed later, at zero frequency $Z_{\mathrm{m}}=R_{\mathrm{m}}$ and Eq. [7] reduces to

$$
\Delta V . \cong \frac{1}{2} V_{\mathrm{m}}(0)
$$


Therefore, under the assumptions indicated, the membrane voltage can be quantitatively related to the extracellular dc voltage imposed between two adjacent nodes.

Maxwell (14) and Rush (21) found that the voltage resulting from a point source of current applied to an anisotropic medium is

$$
V=\frac{I_{0}}{2 \pi}\left[\frac{Z_{1} Z_{2} Z_{3}}{Z_{1} x^{2}+Z_{2} y^{2}+Z_{3 \Sigma^{2}}}\right],
$$

where $I_{0}$ is the total current delivered in amperes; $Z_{1}, Z_{2}$, and $Z_{3}$ are the specific impedance in ohm-cm in the longitudinal, transverse. and depth directions respectively; and $x, y$, and $\approx$ are the distances in centimeters from the current electrode in the longtitudinal, transverse and depth directions respectively. Ranck and BeMent (20) discussed the application of Eq. [9] to the anisotropic dorsal columns.

If the internodal length is assumed to be linearly related to the axis cylinder radius $r$, by a constant $K$, then from Eq. [9] the internodal voltage $\Delta V_{\mathrm{e}}$ can be expressed as

$$
\begin{aligned}
& \Delta V_{\mathrm{e}}=\frac{I_{0}}{2 \pi}\left[Z_{1} Z_{2} Z_{3}\right]^{ \pm}\left\{\left[\frac{1}{Z_{1} x^{2}+Z_{2} y^{2}+Z_{3} \Xi^{2}}\right]^{\frac{1}{2}}-\right. \\
& {\left[\frac{1}{Z_{1}(x+K r)^{2}+Z_{2} y^{2}+Z_{3 z^{2}}}\right] \text { ? }}
\end{aligned}
$$

When Eq. [10] is combined with Eq. [8] and solved for $I_{n}$, the result is

$$
\begin{aligned}
& I_{\mathrm{n}}=\frac{\pi V_{\mathrm{m}}(0)}{\left[Z_{1} Z_{2} Z_{3}\right]^{3}}\left\{\left[\frac{1}{Z_{1} x^{2}+Z_{2} y^{2}+Z_{3 \Sigma^{2}}}\right]^{\frac{1}{2}}-\right. \\
& \left.\left[\frac{1}{Z_{1}(x+K r)^{2}+Z_{2} y^{2}+Z_{3 w^{2}}}\right]^{3}\right\}^{-1}
\end{aligned}
$$

If one assumes that about $15 \mathrm{mv}$ is necessary to trigger an action potential, that no accommodation occurs, and that $Z_{2}=Z_{3}=3 Z_{1}$ where $Z_{1} \cong 200 \mathrm{ohm}-\mathrm{cm}$, then

$$
I_{0}=79 \times 10^{-6}\left\{\left[\frac{1}{x^{2}+3\left(y^{2}+z^{2}\right)}\right]^{1}-\left[\frac{1}{(x+K r)^{2}+3\left(y^{2}+z^{2}\right)}\right]^{1)}\right\}^{-1} \text {. }
$$

When the stimulating electrode is directly above a node, $x=0$; and if $d^{2}=y^{2}+z^{2}$,

$$
I_{0}=79 \times 10^{-6}\left[\frac{1}{3 d}-\left[\frac{1}{K^{2} r^{2}+3 d^{2}}\right]^{1}\right]^{-1}
$$

where $d$ represents the shortest distance of a fiber from the stimulating electrode. Therefore, Eq. [13] represents the relationship between the 
distance $d$ and the dc current $I_{0}$ required to stimulate a particular fiber for the stated assumptions. This relationship becomes fully quantitative when a particular fiber radius $r$ and a suitable $K$ are assumed.

\section{An Analysis of the Factors and Assumptions which Make the Model Quantitative}

Many of the anatomical and physiological factors that can influence the electrical stimulation and conduction characteristics of myelinated nerve fibers are known. The purpose of this section is to examine in greater detail those factors which contribute to the quantitative nature of the model developed. The next section compares the experimental data with the relations predicted by the model, based upon the specific values of the factors discussed below.

1. The Specific Impedance of the Medium. The impedance of the tissuc or medium in which the fiber resides determines the electrical field and current flow from a remote stimulating electrode. The specific impedance of the anisotropic dorsal columns is about three to six times as large in the transverse and depth directions as it is in the longitudinal direction (20). The longitudinal specific impedance of $200 \mathrm{ohm}-\mathrm{cm}$ actually used in the model is a compromise value from their data and neglects the slight frequency dependance of the impedance since their maximal phase angle is only about $7 \mathrm{deg}$, with smaller or unmeasurable angles at frequencies lower than $5,000 \mathrm{~Hz}$.

2. The Membrane Depolarization Necessary for Firing. The change in the membrane potential required to trigger an action potential is generally considered to be 10-20 mv. Tasaki (23) stated that the threshold level for a peripheral myelinated fiber is about $15 \mathrm{mv}$ above the resting potential for short (e.g., $50 \mu \mathrm{sec}$ ) pulses. Coombs, Curtis, and Eccles (5) found the threshold depolarization for the initial segment of motoneurones to be in the range of 5-18 mv. Therefore, a somewhat arbitrary, but reasonable value of $V_{\mathrm{m}}=15 \mathrm{mv}$ is used in the model.

3. The Assumption that $\mathrm{Rm}=4 l\left(\mathrm{nr}_{\mathrm{p}}+\mathrm{r}_{\mathrm{i}}\right)$. The constant 4 can be considered as representing the ratio of nodal membrane resistance to axoplasmic resistance since $n r_{\mathrm{e}} \ll r_{\mathrm{e}}$. If the symbol $R R$ is used to denote such a resistance ratio, then for $K=400$ the equation

$$
R R=\tau /\left(800 \rho w c_{\mathrm{m}}\right)
$$

can easily be derived, where $\tau$ is the nodal time constant, $\rho$ is the axoplasmic resistivity, $w$ is the nodal length and $c_{\mathrm{m}}$ is the nodal capacitance per unit area. Each of these factors is discussed now so that Eq. [14] can be used to estimate $R R$ for mammalian myelinated fibers on the basis of 
reasonable data. The nodal time constant $\tau$ is estimated to be between 75 and $125 \mu \mathrm{sec}$ from the strength-duration data of Figs. 6 and 7 of the previous paper (2a) and from the frequency dependence of the impedance found by Ranck and BeMent. The axoplasmic resistivity $\rho$ is not known with great precision. However, the resistivity of cytoplasm is $1.5-3$ times that of interstitial fluid in all cells studies (17), and the resistivity of cerebrospinal fluid is about $60 \mathrm{ohm}-\mathrm{cm}$ (8). The nodal length $w$ of cut dorsal column fibers is $0.5-1.5 \mu$ (3). The nodal capacitance $c_{\mathrm{m}}$ is not known with great precision. However, most biological membranes are thought to have capacitances of the order of $1 \mu \mathrm{F} / \mathrm{cm}^{2}$, although Tasaki (23) estimated $c_{\mathrm{m}}$ to be $3-7 \mu \mathrm{F} / \mathrm{cm}^{2}$ for frog sciatic nerve fibers, Eccles (7) found mammalian motoneuron membrane capacitances of about $6 \mu \mathrm{F} / \mathrm{cm}^{2}$, and Lux and Pollen (12) obtained membrane capacitances of $1.5-5.0 \mu \mathrm{F} / \mathrm{cm}^{2}$ for large Betz cells.

The value $R R=4$ used in the development of Eq. [8] seems reasonable for mammalian myelinated fibers since $R R=4.16$ when $\tau=100 \mu \mathrm{sec}, \rho=$ $150 \mathrm{ohm}-\mathrm{cm}, w=1.0 \mu$, and $c_{\mathrm{m}}=2 \mu \mathrm{F} / \mathrm{cm}^{2}$. Certainly the lack of precise data for the factors in Eq. [14] affects the credibility of any value of $R R$ selected.

4. The Nodal Time Constant. The time constant of the nodal membrane certainly influences the relation between the stimulus pulse duration and the amount of current required to stimulate a myelinated fiber, as can be seen from the strength-duration relations of Figs. 6 and 7 of the previous paper $(2 a)$. Therefore, in order to compare the experimental results with the values predicted by the quantitative model, the dc stimulating current predicted by Eq. [13] has to be adjusted to correspond to the actual experimental situation where the stimulating current pulse was $50 \mu \mathrm{sec}$ long. This adjustment is made by assuming that nodal depolarization changes exponentially with time constant $\tau$ as the $50 \mu \mathrm{sec}$ current pulse is imposed. The quantitative model specifically assumes that $\tau=100 \mu \mathrm{sec}$ which is about the nodal membrane time constant. Therefore, when the stimulus duration $t=50 \mu \mathrm{sec}, \exp (-t / \tau) \cong 0.4$, which means that the equivalent stimulating current for a $50-\mu \mathrm{sec}$ pulse duration is about 2.5 times the dc stimulating current predicted by Eq. [13].

5. The Internodal Length. The results shown in Fig. 5 of the previous paper (2a) indicate that it is possible to measure with electrophysiological methods what may be the internodal length of a fiber. The model assumes that the internodal length is about 200 times the axis cylinder diameter ( $K=400$ ) from the anatomical data of Hess and Young (9) and Bodian $(2 b)$. Unfortunately, when the conduction velocity for the fiber of Fig. 5 of the previous paper is calculated from an apparent internodal length of 625 
$\mu$, a factor of 0.8 to relate the axis cylinder diameter to the total fiber diameter, and Hursh's factor of 6 , the calculated conduction velocity of 23 $\mathrm{m} / \mathrm{sec}$ is less than one-half the conduction velocity actually measured for that fiber, $53 \mathrm{~m} / \mathrm{sec}$. For four other fibers investigated the differences between the calculated and measured conduction velocities, relative to the measured conduction velocity, are $-1,-7,+17$, and $-40 \%$, where the longitudinal stimulation properties are obtained for one period or more. Therefore, a much larger study of the longitudinal stimulation properties of these myelinated fibers is necessary to check by electrophysiological means the assumption of $K=400$ based upon the anatomical data.

6. The Fiber Diameter. There is no method presently available which allows one to obtain the actual diameter of a fiber from which one is recording in wivo with the preparation and method used here. However, an analysis of the fibers shown in an electron micrograph of the cervical region of the cat dorsal columns shows a median axis cylinder diameter of about $4 \mu$, with a median outside diameter of about $5 \mu$. The median conduction velocity for all of the fibers presented in this study is about 37 $\mathrm{m} / \mathrm{sec}$; therefore. Hursh's factor of 6 predicts a median outside fiber diameter of about $6 \mu$. In view of the errors associated with the preparation of the electron micrographs and with the determination of the conduction velocities, there seems to be good agreement between the anatomically and the physiologically derived median fiber diameter.

7. Parameter Variations. The values of de stimulating current predicted by Eq. [13] can be altered by parameter variations in two distinct ways. Variations in the values of items 1,2 , and 3 result in changes in the multiplicative constant, $79 \times 10^{-6}$. Variations in the factor $K$ relating fiber diameter to internodal length result in nonlinear changes in the predicted values of stimulating current. Figure 3 indicates the sensitivity of the current-distance relationship predicted by the model for other values of $K$ since the broken curves indicate the predicted relationship for a 7.5- $\mu$ -diameter fiber when $K=300$ and $K=500$, relative to the solid curve for $K=400$.

The factors and assumptions just discussed are considered to be of principal importance in the development of a quantitative model to describe a relationship between stimulus intensity and distance from the stimulating electrode. However, because of the uncertainty associated with many of the factors involved, the exact values assumed in the model may be quite untrue. Nevertheless, these values seem to be the most reasonable ones available based on the data and relationships known at the present time. The next section compares the experimental data with the relations predicted by the model under the assumptions discussed above. 


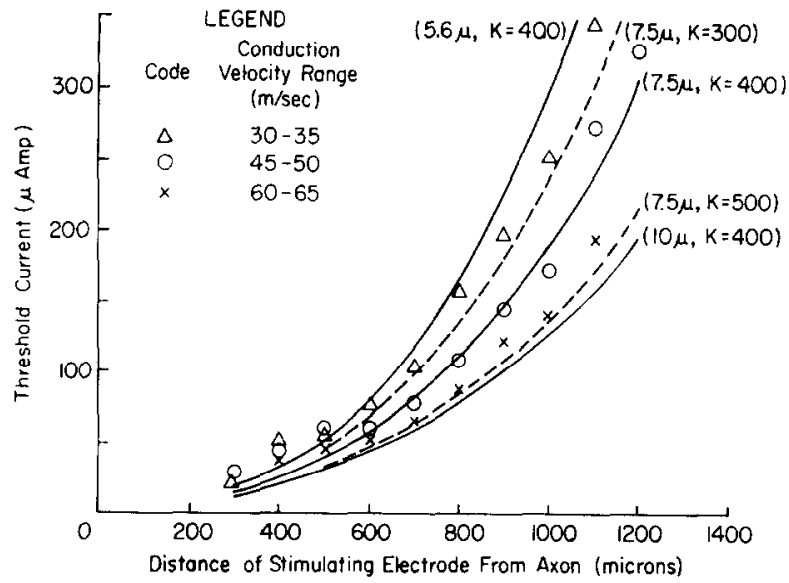

Fig. 3. A comparison of experimental data with a few theoretical relations predicted by Eq. [13]. The solid curves are derived from the equation with $K=400$ and three values of fiber diameter. The broken curves assume a 7.5- $\mu$ fiber diameter and two other values of $K$. The individual points indicate averaged experimental data for three distinct ranges of conduction velocity shown in the legend. These points are from data obtained in the study described in the previous paper $(2 a)$.

\section{A Comparison of the Experimental Data with the Model Predictions}

The solid curves of Fig. 3 show the current-distance relationships predicted by three different fiber diameters for $K=400$. The individual points represent the averaged stimulation data for fibers separated into three distinct ranges of conduction velocities and were obtained from the data associated with the previous paper. When the conduction velocity is assumed to be related to the fiber diameter by Hursh's factor of 6 , there is reasonable agreement between the curves predicted by the model and the experimental data for electrode distances of $600 \mu$ or greater. Furthermore, for these larger distances the general shape of the curves predicted by the model seems to agree with the shape of curves that could represent the data points.

The model also predicts that curves which correspond to a doubling of the diameter of a fiber should compare with clata points associated with a loubling of the conduction velocity. However, Fig. 3 indicates that such a correlation between the fiber diameter as represented in the model and the conduction velocity as represented by the experimental data is not as good as one might wish. Therefore, the model appears to have at least one reasonably important shortcoming.

The values of stimulating current predicted by the model seem to depart 
from the experimental values to an increasingly greater extent as the electrode distance becomes less than $500 \mu$. Such a lack of agrcement at the smaller electrode distances is not surprising because $(a)$ the errors associated with the acquisition of the experimental data have been shown to be largest at the smaller distances and (b) Eq. [9], which describes the voltage developed in an anisotropic medium from a point source of current, becomes increasingly less valid at smaller electrode distances. When Eq. [9] is rewritten with $Z_{1}=200 \mathrm{ohm}-\mathrm{cm}, Z_{2}=Z_{3}=3 Z_{1}$, and $d^{2}=y^{2}+z^{2}$, the result is

$$
V=9.55 \times 10^{3} I_{0} /\left[x^{2}+3 d^{2}\right]^{\frac{1}{2}} \text {. }
$$

The quantity $V / I_{0}$ from Eq. [15] is plotted in Fig. 4 with respect to $x$, the longitudinal distance from the current source. The parameter $d$ is the net distance of the fiber from the stimulating electrode. The figure indicates that for $d$ 's less than about $400 \mu, \mathrm{Eq}$. [9] violates rather significantly the assumption of a linear voltage change with distance in the ir direction.

There are other reasons why the model may be inaccurate at smaller electrode distances. In the usual stimulation situation, the stimulating electrode is not directly above the stimulated node. In fact, the median distance of the stimulating electrode from the stimulated node in the $x$ direction can be shown to be about $150-400 \mu$, depending upon the internodal length of the fiber. Furthermore, the stimulating electrode diameter is large $(100 \mu)$ when compared with the usual nodal width $(0.5-1.5 \mu)$. However, at distances greater than 500-600 $\mu$ these factors tend to become less important and may even be negligible.

We have not directly tested the assumption that central myelinated fibers have saltatory conduction properties that follow the same laws as for peripheral myelinated fibers. However, we feel that this assumption is

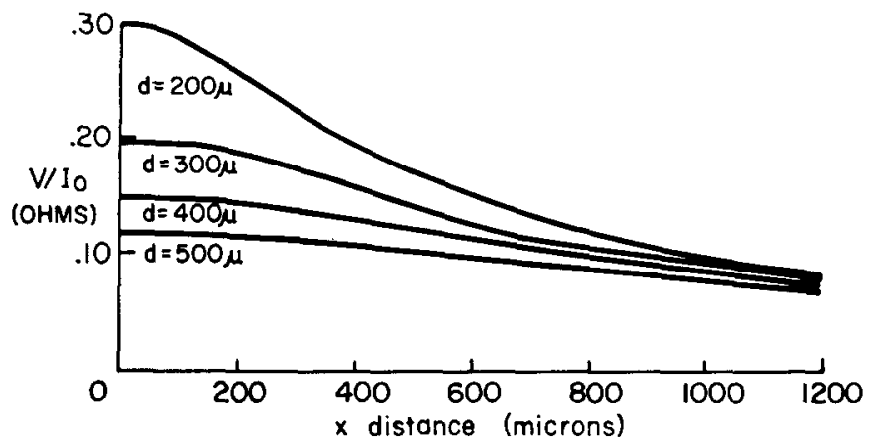

Fig. 4. Equation [i5] plotted in terms of $l / I_{0}$ versus $x$ for various values of $d$. 
valid based upon similarities hetween peripheral and central myelinated fibers with respect to their nodal and internodal anatomy (4), the regularity of internodal distances, and the variation in threshold exhibited by the longitudinal stimulation data. The approximations used to establish the basic equations for the model-infinite internodal membrane resistance. zero internodal membrane capacitance and a linear extracellular potential gradient along the fiber-are certainly not strictly correct. However, it appears unlikely that minor changes in such approximations would result in major changes in either the absolute values or the shape of the curves.

We do not have precise values for all of the terms that are necessary for a fully quantitative model. However, we have indicated that enough is known about each of these factors so that a range of values can be assumed. Furthermore, agreement between the experimental stimulation data and the model is achieved with values comfortably within these ranges. Therefore. given the basic assumption of saltatory conduction, the changes in the predicted stimulation characteristics due to a relatize change in some parameter is clear. Thus, the moclel allows us to predict electrical stimulation characteristics for situations other than those for which we have direct experimental clata. in particular, for stimulation in areas with other specific impedances and over wider ranges of fiber conduction velocities.

\section{Practical Applications of the Data and Model, with Limitations}

In one sense the experimental data presented in Fig. 3 (and Fig. 4 of the previous paper) represent a fairly restrictive set of stimulation circumstances because they were obtained with a $50-\mu \mathrm{sec}$ rectangular cathodal current pulse delivered through a monopolar $100-\mu$ diameter surface electrode into an anisotropic medium from a distance of $300-1200 \mu$. Therefore, a certain degree of caution should be employed in any attempt to generalize these results to other systems of clectrorles, stimuli, excitable clements, and biological media. The purpose of this section is to indicate some practical applications, extensions, and limitations of the data and the model and can be considered as an "instruction manual' for the use of Figs. 3 and 5 , and Fig. 7 of the previous paper $(2 a)$.

1. Distances Less Than $500 \mu$. One has to be particularly cautious when interpreting or extencling the clata and the model for electrode distances less than about $500 \mu$ because each is known to be increasingly inaccurate for smaller and smaller distances. However, the clata do indicate that the threshold stimulation current is dependent upon the distance of the electrode frum the fiber. Furthermore, the data from the longitudinal stimulation studies give some indication of the dependence of the stimulating current upon the position of the stimulating electrode relative to the nodes 
of a fiber. Therefore, it seems reasonable to generalize from $(a)$ the extreme values, the mean, and the standard deviation of the raw data, and (b) the longitudinal stimulation properties, that about 10-70 $\mu$ amp of 50$\mu \mathrm{sec}$ cathodal current is required to stimulate fibers about $300 \mu$ from a $100-\mu$ diameter surface stimulating electrode. Similarly about 25-100 $\mu \mathrm{amp}$ seems necessary to stimulate fibers about $500 \mu$ from the same electrode. The relatively larger experimental errors that occur for these smaller distances do not permit any definite conclusions concerning the effect of conduction velocity upon the threshold stimulation current.

2. Distances from 500 to $1200 \mu$. For stimulating electrode distances greater than $500-600 \mu$, the amount of stimulating current can also be related to the conduction velocity of a fiber, whereas the position of the nodes relative to the stimulating electrode becomes of less importance. The data for these larger distances imply that a $150 \mu$ amp current will generally stimulate $60 \mathrm{~m} / \mathrm{sec}$ fibers located $800-1200 \mu$ from the stimulating electrode, and $30 / \mathrm{sec}$ fibers located $600-900 \mu$ from the stimulating electrode. The respective limiting values suggested here are based primarily upon the mean values and standard deviations obtained from Table 1 of the previous paper $(2 a)$.

3. Extensions to Other Conduction Velocities. The model can be extended to other ranges of conduction velocities with the stipulation that the limits of error are increased appropriately, since no experimental verification exists from our data for conduction velocities less than about 25 $\mathrm{m} / \mathrm{sec}$ or greater than about $65 \mathrm{~m} / \mathrm{sec}$. Such extensions at least give an idea of the order of magnitude of the stimulating currents required and the distribution of fibers that might be stimulated by a given current.

4. Extensions to Other Regions of Neural Tissue. No effort was made in these studies to investigate the electrical stimulation properties of single myelinated fibers located in other regions of the central nervous system. However, there appear to be no obvious reasons why the model developed for the electrical stimulation of dorsal-column fibers could not be generalized to include the electrical stimulation of myelinated fibers in other regions, once the impedance of the other tissue is determined and the model altered accordingly.

Impedance measurements of central white matter have been obtained only from the dorsal columns (20) and the internal capsule (15). The lack of precision associated with both of these measurements seems sufficient to explain any differences in the values obtained. Therefore, until more precise measurements are made we shall assume that the impedance of these two regions of white matter is the same.

Figure 5 illustrates current-distance relations obtained from the model for myelinated fibers of different conduction velocities located in a medium 


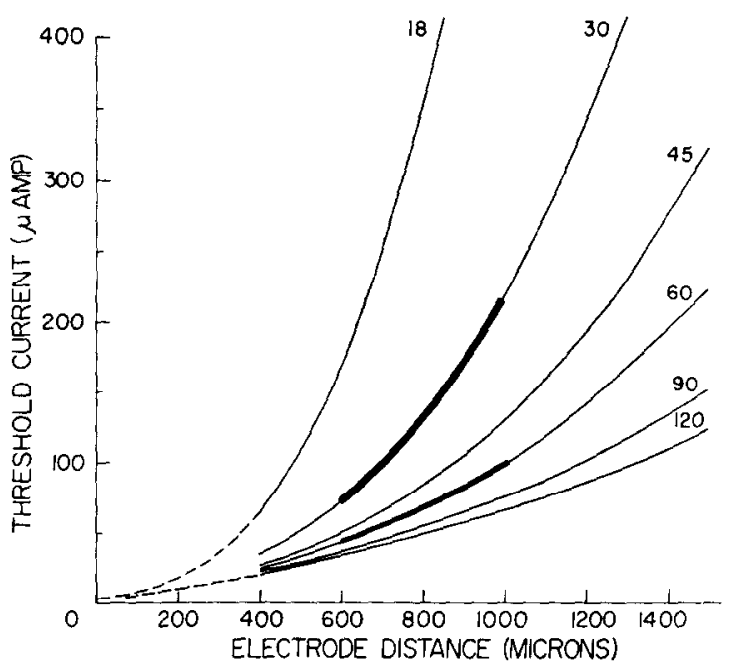

FIG. 5. Current-distance relations predicted from eq. [11] for the stimulation of myelinated fibers in an nonhomogeneous, isotropic medium where $Z_{1}=Z_{2}=Z_{3}=250$ $\mathrm{ohms} / \mathrm{cm}$ in all directions. The stimulus is again a $50 \mu \mathrm{sec}$ rectangular monopolar cathodal current pulse and the other constants in the equation are the same as assumed previously. The number beside each curve is the conduction velocity predicted from the particular fiber diameter assumed for each curve. The darkened portions on two of the curves represent the limits of the dorsal column experimental data translated into this different nonhomogeneous, isotropic medium.

of $250 \mathrm{ohm} / \mathrm{cm}$ specific resistance (19). Here again the stimulus is assumed to be a $50-\mu \mathrm{sec}$ rectangular monopolar current pulse originating from a cathodal electrode located $400-1500 \mu$ from the site of stimulation. The conduction velocity range indicated $(15-120 \mathrm{~m} / \mathrm{sec})$ corresponds to a range of fiber diameter from about 3 to about $20 \mu$, which includes most of the myelinated fibers usually found in the central nervous system. The relations predicted by the model have been given for distances up to $1500 \mu$ even though the underlying experimental data were obtained only for distances up to about $1200 \mu$. However, we feel that the form and basic relations represented by the curves should not be significantly different at these extended distances.

The physiological mediums represented in Figs. 3 and 5 correspuid more or less to the extremes of specific resistances likely to be encountered in the central nervous system, i.e., Fig. 3 is for a homogeneous anisotropic medium with a specific resistivity of about $600 \mathrm{ohm}-\mathrm{cm}$ in two directions, and Fig. 5 is for a homogeneous isotropic medium with a specific resistance of about $250 \mathrm{ohm}-\mathrm{cm}$ in all directions. Therefore, stimulation in regions of other resistivities may require some interpolation hetween the two figures. 
However, note that Figs. 3 and 5 are not actually very different when one is supcrimposed on the other.

5. Boundary Effects. The experimental data were obtained far enough from the border of the dorsal columns so that the effects of adjacent gray matter of different impedance could be ignored. The model also assumed that the dorsal column medium was of infinite extent. However, since the results of Figs. 3 and 5 do not differ by nuch. there should not be very large effects on predicted stimulation currents because of adjacent areas of gray or white matter and such effects can be guessed by interpolating between the two figures. However, one would expect a significant difference for a situation where the stimulating electrode was not located on a surface exposed to air. Thus, if the stimulating electrode is in the middle of a tissue media, about twice as much current should be required to stimulate at a given distance as when the electrode is on the surface. If cerebrospinal fluid (resistivity about $60 \mathrm{ohm}-\mathrm{cm}$ ) is near the region to be stimulated, much of the current will flow into this low resistance fluid. For instance, if a stimulating electrode is on a gray matter surface covered with a large volume of cerebrospinal fluid or physiological saline solution, about four times as much current will flow in the fluid as in the tissue. Therefore, about five times as much current should be required to stimulate from a fluid covered surface as from a dry surface.

6. Stimulus Curront Duration. The normalized strength-duration data shown in Fig. 7 of the previous paper (2a) allow one to transform the threshold stimulation data obtained for a $50-\mu \mathrm{sec}$ rectangular stimulating pulse into equivalent data for other rectangular pulse durations. For example, the same fiber should take about two-thirds as much $100-\mu \mathrm{sec}$ stimulating current and about one-half as much $150-\mu \mathrm{sec}$ stimulating current as is required for stimulation with a $50-\mu \mathrm{sec}$ stimulating current, all other factors being equal. Thereforc, the ranges of stimulus current and electrode distances predicted previously can be translated into equivalent ranges for other stimulus durations.

7. Stimulus Polarity. We have indicated that stimulating myelinated fibers from a monopolar anode require from 3.2 to 7.7 times as much current as stimulating from a monopolar cathode. Similar findings have been reported for gray matter $(22)$.

8. Electrode Siac. The actual diameter of the uninsulated tip of the stimulating electrode should influence the current density at the site of stimulation only when the distance to the site of stimulation is of the same magnitude as the diameter of the electrode.

9. Other Electrode Types. We do not have experimental or analytic results for bipolar or concentric electrodes. Presumably, such results could be obtained by the same methods employed for the monopolar case. 


\section{General Comments on Extracellular Electrical Stimulation of the Central Nervous System}

It is certain that myelinated fibers are often stimulated directly in extracellular stimulation of the central nervous system. It is almost equally certain that other elements are also stimulated directly. The data of Stoney, Thompson, and Asanuma (22) indicate that cell bodies or something in the vicinity of cell bodies may be the element directly stimulated. The greater ease with which many neocortical neurons are stimulated by surface anodal currents than with surface cathodal currents (18) also seems to indicate that something in the vicinity of cell bodies is being stimulated. Chronaxies for central stimulation of gray natter are often about 200-400 $\mu \mathrm{sec}(1,22)$ which is difficult to explain since myelinated fibers have chronaxies of about $100 \mu \mathrm{sec}$, whereas cell hodies and initial segments of proximal dendrites should have chronaxies greater than 1 msec. Perhaps some central nodes of Ranvier have longer time constants than those found for dorsal column fibers. If chronaxies were either about $100 \mu \mathrm{sec}$ or greater than $1 \mathrm{msec}$ at least one could be somewhat certain of what is heing stimulated. Note that MacLean and Ploog (13), in a study of penile erection, found that in some electrode locations trains of $1 \mathrm{msec}$ pulses were most effective and in other locations trains of $10-50-\mu \mathrm{sec}$ pulses were most effective. Doty (6) found that stimulation of cat neocortex to give a conditioned response resulted in a chronaxie of about $1 \mathrm{msec}$.

Since data on strength-duration relations for central stimulation are generally not available, we recommend obtaining and publishing strengthduration curves routinely.

\section{References}

1. Arfles, M. 1967. Excitability of EFG "synchronizing" and "desynchronizing" neurones in the thalamus and the brain-stem of the cat. I. The applicability of strength-duration determinations and conditioning-test technique. Electroencephalog. Clin. Neurophysiol. $23: 1$ (1-24.

2a. BeMext, S. L., and J. B. Raxik, JR. 1969. A quantitative study of electrical stimulation of myelinated fibers. Exptl. Nourol. 24 : (in press).

26. Bondx, D. 1951. A note on nodes of Ranvier in the central nervous system. $J$. Comp. Nourol. $94: 475-483$.

3. Bunge, R. P., M. B. Bunge, and H. R1s. 1960. Electron microscopic study of demyelination in an experimentally induced lesion in adult cat spinal cord. $J$. Biophys. Biochem. Cytol. 7: 685-696.

4. Benge, R. P. 1968. Glial cells and the central myelin sheath. Physiol. Rer. 48: 197-251.

5. Coombs, J. S., D. R. Curtis, and J. C. Eccles. 1957. The generation of impulses in motoncurones. J. Physiol. London 139 : 232-249.

6. Doty, R. W., L. T. Ru'tledge, and R. M. Larson. 1956. Conditioned reflexes cstablished to electrical stimulation of cat cerebral cortex. J. Neurophysiol. 19 . 401-415. 
7. Eccles, J. C. 1957. "The Physiology of Nerve Cells." John Hopkins Press, Baltimore.

8. Geddes, L. A. and L. E. Baker. 1967. The specific resistance of biological material-A compendium of data for the biomedical engineer and physiologist. Med. Biol. Enyr. 5: 271-293.

9. Hess, A. and J. Z. Young. 1949. Correlation of internodal length and fibre diameter in the central nervous system. Nature 164: 490-491.

10. Hodgkix, A. L. and A. F. Huxley. 1952. A quantitative description of membranc current and its application to conduction and excitation in nerve. J. Physiol. London 117: 500-544.

11. Hodgkin, A. L. and W. A. H. Rushton. 1946. The elcetric constants of a crustacean nerve fibre. Proc. Roy. Soc. London, Scr. B. 133 ; 444-479.

12. Lux, H. D. and D. A. Pollen. 1966. Electrical constants of neurons in the motor cortex of the cat. J. Neurophysiol. $29: 207-220$.

13. Maclean, P. D. and D. W. Ploog. 1962. Cerebral representation of penile erection. J. Ncurophysiol. 25 : 20-55.

14. Maxwfll, J. C. 1891. "A Treatise on Electricity and Magnetism." 3rd ed., Section 297-302. Dover, New York.

15. Nicholson, P. W. 1965. Specific impedance of cerebral white matter. Exptl. Neurol. $13: 386-401$.

16. Patlak, C. S. 1955. Potential and current distribution in nerve: The effect of the nerve sheath, the number of fibers, and the frequency of alternating current stimulation. Bull. Math. Biophyss. 17: 287-307.

17. PaUly, H. and L. Packrer. 1960. The relationship of internal conductance and membrane capacity to mitochondrial volume. J. Biophys. Biochesn. Cytol. 7: 603-612.

18. Purpura, D. P. and J. G. McMurtry. 1965. Intracellular activities and evoked potential changes during polarization of motor cortex. J. Neurophysiol. 28: 166-185.

19. Ranck, J. B. 1963. Specific impedance of rabbit cerebral cortex, Exptl. Ncurol. 7 : $144-152$

20. Ranck, J. B. and S. L. BeMent. 1965. The specific impedance of the dorsal columns of cat: An anisotropic medium. E.rptl. Neurol. 11: 451-463.

21. Rush, S. 1962. Methods of measuring the resistivities of anisotropic conducting media in situ. J. Res. Natl. Bur. Stand. C66: 217-222.

22. Stoney, S. D., W. D. Thompson, and H. Asancma. 1968. Excitation of pyramidal tract cells by intracortical microstimulation: The effective extent of the stimulating current. J. Neurophysiol. 31: 659-669.

23. TASAKI, I. 1959. Conduction of the nervous impulse. In "Handbook of Physiology" Sec. 1. "Ncurophysiology" 1. Field [El.], American Physiological Society, Washington.

24. TAYLOR, R. E. 1952. The distribution of membrane current in nerve with longitudinal linearly increasing applied current. Bull. Math. Biophys. 14 : 265-292. 